Gynäk. Rdsch. 1982;22:I-VIII

\title{
Contents, Vol. 22, 1982
}

\section{Nr. 1 Originalarbeiten}

Hormonaktivität einiger Brenner-Tumoren

Jalûvka, V.; Kratzsch, E 1

Über die zyklische Behandlung klimakterischer Beschwerden mit genuinen Östrogenen

Bartl, W.; Kemeter, P.; Friedrich, F.; Seidl, A

Zervikale Gravidität - Diagnose und Therapie

Goessens, L.; Leucht, W.; Müller, E 49

Kurzfassungen von Zeitschriftenartikeln

Hochdosierte Gestagen-Langzeittherapie beim Korpuskarzinom. Einfluss auf die Überlebenszeit

Fournier, D. v.; Kubli, F.; Bauer, M.; Weber, E 58

Das primäre Tubenkarzinom. Eine klinische Studie an 37 Patientinnen

Engeler, V.; Reinisch, E.; Schreiner, W.E 59

Objektiv nachgewiesene Abnahme von vasomotorischen Hushes in der Postmenopause unter Plazebo- und oraler Medroxyprogesteronazetat-Therapie

Albrecht, B.H.; Schiff, I.; Tulchinsky, D.; Ryan, K.J 61

Zervixkarzinom: ein Versuch, die Behandlung zu individualisieren. Ergebnisse einergemeinsamen 20-Jahres-Studie

Zander, J.; Baltzer, J.; Lohe, K.J.; Ober, K.G.; Kaufmann, C

Vergleich von Plasma-Prostaglandin-Konzentrationen am Termin und bei Frühgeburt

Sellers, S.M.; Mitchell, M.D.; Bibby, J.G.; Turnbull, A.C 65

Prognostische Bedeutung der negativen Histologie nach Hysterektomie des präoperativ

intrakavitär bestrahlten Korpuskarzinoms im Stadium I

Cheung, A.Y.C

66

Dopaminergic Mechanisms and Luteinizing Hormone (LH) Secretion. II. Differential Effects of Dopamine and Bromocriptine on LH Release in Normal Women

Martin, W.H.; Rolog, A.D.†; Kaiser, D.L.; Thorner, M.0 67

Die Verwendung der transkutanen Nervenstimulation zur Verminderung des Wehen-

schmerzes

Nesheim, B.-1 69

Das Plazentainsuffizienzsyndrom. Ein klinisches Konzept

Wulf, K.H $\quad 70$

Fortbildung

Neues zur Östrogen- und Gestagentherapie

1. Nutzen und Risiko der Östrogentherapie Östrogen-Rezeptor-Wechselwirkung und ihre

klinische Bedeutung

Bergink, E.W 73

IV

Inhaltsverzeichnis 
Besondere Aspekte der Östrogentherapie

Rauramo, L 74

Hormonbehandlung in der Postmenopause. Eine Nutzen-Risiko-Abwägung

Plotz, E.J 75

2. Orale Kontrazeption mit einem neuen Gestagen von Organon Entwicklung und

pharmakologische Eigenschaften von Org, 2969

Vossenaar, T 76

Ein neues Gestagen in der konischen Pruning

Nevinny-Stickel, J 77

Orale Kontrazeptiva und Fettstoffwechsel

Samsioe, G 78

Biochemische Befunde und klinische Erfahrungen mit einem neuen Kontrazeptivum bei

Jugendlichen

Mall-Haefeli, M

Ankündigung 80

Nr. 2 Originalarbeiten

Le conseil génétique et le diagnostic antenatal en cas de grossesse gémellaire

Pescia, G.; Nguyen The, H 81

Postoperative Strahlentherapie der epithelialen Ovarialkarzinome

Kogelnik, H.D.; Szepesi, T 88

Fruchtwasserembolie. Ubersicht und Hinweise auf eine vorwiegend pulmonal disseminierte intravasale Gerinnung

Schmid, F.; Zimmermann, A.; Cottier, H 96

Erfahrungen mit einem kulturellen Schnelltest zum Candidanachweis in einer gynäkologischen Ambulanz

Grünberger, W.; Pateisky, N.; Husslein, P.; Schmid, R

Behandlung von Portioektopie, Zervikalkanalpolyp und postoperativem Granulom mit Solcogyn (mit 2 Farbtafeln)

Lurie, D 113

Kurzfassungen von Zeitschriftenartikeln Urologische Komplikationen der operativen

Behandlung des Zervixkarzinoms mittels Oka-

bayashi-Technik

Bostofte, E.; Serup, J 118

Östrogenmedikation bei Frauen mit Endometriumkarzinom. Eine retrospektive Vergleichsstudie aus dem Radiumhemmet

Öbrink, A.; Bunne, G.; Collén, J.; Tjernberg, T

Ergebnisse der Gestagentherapie von Endometriumkarzinomen unter besonderer Berücksichtigung prätherapeutischer In-vitro-Testergebnisse

Schindler, C; Schober, J

121

Zur Frage der sogenannten konservativen Behandlung des atypischen Zervixepithels

Burghardt, E 122

Ursprung und Stoffwechsel des Kortisols beim fetalen Rhesusaffen in der Spätschwangerschaft

Mitchell, B.; Serón-Ferré, M.; Hess, D.; Jaffe, R.B 124

Inhaltsverzeichnis $\mathrm{V}$

Klinische Erfahrungen mit neun in vitro fertilisierten Schwangerschaften 
Wood, C; Trounson, A.; Leeton, J.; Mckenzie Talbot, J.; Buttery, B.; Weeb, J.; Wood, J.; Jessup, D 126

Prolactinstimulierter ovarieller Androgenmetabolismus

Polan, ML.; Berman, H.R 127

Einfluss von Hexoprenalin auf das Verhältnis Lezithin/Sphingomyelin sowie auf die

Druck/Volumen-Kurven fetaler Lungen beim Kaninchen

Lipshitz, J.; Broyles, K.; Hessler, J.R.; Whybrew, W.D.; Ahokas, R.A.; Anderson, G.D. 129 Die endokrine Regulation der Blutzirkulation des nichtschwangeren Uterus: eine Übersichtsarbeit

Resnik, R $\quad 130$

Der Wert der Laparoskopie in der Verlaufskontrolle des fortgeschrittenen Ovarialkarzinoms

Ozols, R.F.; Fisher, R.I.; Anderson, T.; Makuch, R.; Young, R.C 132

Das Endometriumkarzinom: eine Abweichung von der normalen Differenzierung der Endometriumzellen

Satyaswaroop, P.G.; Mortel, R

Die Bestimmung von Plazenta-Protein 5, Plazenta-Laktogen und schwangerschaftsspezifischem ßi-Glykoprotein im mittleren Schwangerschaftsdrittel und deren Aussage für den Schwangerschaftsausgang

Salem, H.T.; Lee, J.N.; Seppälä, M.; Vaara, L.; Aula, P.; Al-Ani, A.T.M.; Chard, T. .135 Das

Vorkommen von schwerer Präeklampsie bei Müttern und Schwiegermüttern von präeklamptischen Schwangeren

Sutherland, A.; Cooper, D.W.; Howie, P.W.; Liston, W.A.; McGillivray, J 137

Freies Östradiol bei Frauen nach der Menopause mit bzw. ohne Endometriumkarzinom Davidson, B.J.; Gambone, J.C.; Lagasse, L.D.; Castaldo, T.W.; Hammond, G.L.; Siiteri, P.K.; Judd, H.L 138

Bei Töchtern von Frauen mit Mammakarzinom fanden sich erhöhte 24-Stunden-Prolaktinwerte und eine teilweise Resistenz des Prolaktins auf die suppressive Wirkung des Dopamins

Levin, P.A.; Malarkey, W.B 140

Der zirkadiane Rhythmus des Cortisols ist postoperativ gestört

Mclntosh, K.; Lothrop, A.; Lee, A.; Jackson, T.; Nabseth, D.; Egdahl, H 142

Fortbildung

III. Fortbildungstagung der II. Universitäts-Frauenklinik Wien

Obergurgl. 1.-7. Februar 1981 Vorwort

Gitsch, E 144

Chirurgische Behandlung gynäkologischer Malignome

Janisch. H 145

Tumorreduktion durch Second-Look-Laparotomie nach kombinierter Radiochemotherapie beim fortgeschrittenen Ovarialkarzinom

Szalay, S.; Janisch, H.; Breitenecker, G.; Szepesi, T 150

Zeitfaktor der Second-Look-Operation nach Chemotherapie und Strahlentherapie fortgeschrittener epithelialer Ovarialkarzinome. Tumorzellkinetische Überlegungen

Szepesi, T.: Kärcher, K.H.; Kogelnik, H.D.; Janisch, H.; Szalay, S.; Breitenecker, G. .154 Staging- und Gradingverfahren bei Ovarialkarzinomen und deren prognostische Aussagekraft 
Bartl. W.; Janisch, H.; Szalay, S.; Szepesi, T.; Tatra, G.; Breitenecker, G 158

Inhaltsverzeichnis

Originalarbeiten

Fruchtwasserpunktion in der Spätschwangerschañ - eine gefährliche Massnahme?

Nezbeda, J.; Salzer, H.; Husslein, P.; Reinold, E 165

Resultate einer Längsschnitt-Studie über die psychische Verarbeitung der Kontrazeption mit

Multiload Cu 250

Rauchfleisch, U 176

1-Jahres-Katamnese einer Längsschnittstudie über die psychische Verarbeitung der

Kontrazeption mit Multiload Cu 250 (Schlussbericht)

Rauchfleisch, U 183

Serumlipoproteine im normalen Menstruationszyklus. Qualitative und quantitative Unter-

suchungen

Brockerhoff, P.; Höckel, M.; Holzer, A.; Schwenzer, E.; Rathgen, G.H 194

Erfahrungen mit einem neuen Präparat, Solcogyn, zur Behandlung von Portioerosionen (mit

2 Farbtafeln)

Seidl, A.; Bartl, W 204

Kurzfassungen von Zeitschriftenartikeln

Zytologische Langzeitstudie bei Trägerinnen von Kupfer-Intrauterinpessaren

Engineer, A.D.; Misra, J.S 210

Der Einfluss von Alter und Parität auf die Menstruation

Chamberlain, G.; Roman, E.; Pharoah, P.; Alberman, E 211

Klimakterische Wallungen: klinische und hormonelle Reaktionen auf Infusionen mit Naloxon

Lightman, S.L.; Jacobs, H.S.; Maguire, A.K.; McGarrick, G.; Jeffcoate, S.L 212

Fertilität nach beidseitig durchgeführter Keilresektion der Ovarien: eine kritische postoperative

Beurteilung von 90 Fallen mit polyzystischem Ovarialsyndrom

Adashi, E.Y.; Rock, J.A.; Guzick, D.; Colston Wentz, A.; Seegar Jones, G.; Jones, H.W., Jr 214

Klinische und hormonelle Studien der ovariellen Hyperthekose

Nagamani, M.; Lingold, J.C.; Gomez, L.G.; Garza, J.R 216

Beitrag zum Problem des operativen Verschlusses von Blasenscheidenfisteln

Ingelman-Sundberg, A 217

Akuter Effekt des Rauchens auf die uteroplazentare Durchblutung bei normotensiven und

hypertensiven Schwangeren

Lehtovirta, P.; Forss, M 219

Pränataldiagnostik - Bericht über 601 Chromosomenuntersuchungen

Taillemite, J.L.; Le Porrier, N.; Joye, N.; Delubac, D.; Colau, J.C.; Van Den Akker, J.;

Portnoi, M.F.; Roux, Ch 220

Klinisches Vorgehen bei verschwundenem Intrauterinpessar

Tadjerouni, A.; Flici, O.; Wauters, G.; Hubinont, P.0 222

Eine neue Technik zur Anwendung tiefer Temperaturen im Cavum uteri

Dziambor, H.; Renziehausen, K.; Marzotko, F.; Könnecke, J.; Krantz, H 223

Zur Klassifikation der Gestosen. Kurze Mitteilung

Kyank, H 225

Fortbildung 
Kontrazeption: Fortbildungsseminar an der I. Universitäts-Frauenklinik. Wien, 30. 10. 1981 Neue Aspekte in der hormonellen Antikonzeption

Huber, J 227

Inhaltsverzeichnis

VII

Indikationen zur Anwendung des Intrauterinpessars

Fischl, F 229

Barrieremethoden als Alternative in der Kontrazeption

Binstorfer, E 230

Nr. 4 Originalarbeiten

Erfahrungen mit Sulproston zum Cervical Priming beim Schwangerschaftsabbruch im 1.

Trimenon

Heinzl, S.; Winkler, C; Allemann, F 233

Blutungen unter «Low-dose»-Heparin und Plazebo (NaCl-Lösung) in der operativen Gynäkologie

Studer-Schär, A 241

Aktuelle Mitteilungen

Nach einer Vasektomie kommt ein Kind! Die Klinik ist verantwortlich. Neuer Gerichtsentscheid in Amerika

Schweisheimer, W 249

Die weibliche Fruchtbarkeit nimmt nach dem 30. Lebensjahr ab. Ergebnisse einer neuen

Studie mit künstlicher Inseminierung

Schweisheimer, W 251

Kurzfassungen von Zeitschriftenartikeln

Östrogen-Serumspiegel beim Ovarialkarzinom in der Postmenopause.

Heinonen, P.K.; Tuimala, R.; Pyykkö, K.; Pystynen, P 253

Prostaglandin E2 vermindert den Effekt von Angiotensin II auf den Blutdruck bei Schwangeren, nicht aber bei Nichtschwangeren

Broughton Pipkin, F.; Hunter, J.C.; Turner, S.R.; O’Brien, P.M.S 254

Orale Kontrazeptiva als Risikofaktor für Präeklampsie?

Bracken, M.B.; Srisuphan, W 256

Ergebnisse zytologischer Untersuchungen von direkt entnommenem Zellmaterial aus dem Uteruskavum mit der Exploret-Fatol

Clocuh, A.P.A.; Peters-Welte, C 257

Hypotonie in der Schwangerschaft: Krankheitswert, Differentialdiagnose, Konsequenzen

Goeschen, K.; Pluta, M.; Meyer-Wilmes, M.; Saling, E 259

Corpus-Luteum-Funktion nach Follikelaspiration zur Eizellgewinnung

Garcia. J.; Jones, G.S.; Acosta. A.A.; Wright, G.L., Jr 261

Die BCG-Impfung in die Portio uterina als unspezifische regionale Immuntherapie in der gynäkologischen Onkologie

Capello. F.; Meli. S 263

Untersuchungen der Muskelaktivität des menschlichen Eileiters in vitro, in bezug auf die

Plasmakonzentrationen von Östradiol und Progesteron; Einfluss des Noradrenalins auf

diese Aktivität

Helm. G.; Owman. C; Sjöberg. N.O.; Walles. B 265

Die Serum-Beta-Lipoproteinwerte in den Monaten vor dem Auftreten einer Gestose und 
ihre Beziehungen zum Cetrimoniumtest

Badin. J.; Barrier. J.; Denne, M.A.; Jacquet, E

Bakteriologische Aspekte entzündlicher Erkrankungen im weiblichen Genitaltrakt

Creatsas. G.K.; Pavlatos. M.P.: Koumantakis, E.; Zolotas, J.: Kaskarelis, D.B 268

VIII Inhaltsverzeichnis

Paradoxe Hypersekretion des Wachstumshormons bei Patientinnen mit atypischer Hyperplasie des Endometriums und Endometriumkarzinom; die Auswirkung der Uterusexstirpation

Ayalon, D.; Peyser, M.R.; Limor, R.; Lessing, J.B.; Ravid, R 270

Fortbildung

Gynäkologie fur die Praxis:

Fortbildungsseminar vom 29. Januar 1982 an der I. Universitäts-Frauenklinik Wien Einführung zu den Themen

Gitsch, E 272

Neues zur gynäkologischen Instrumentenkunde

Gitsch, E 273

Lokale Nachbehandlung nach Hysterektomien, Konisationen und Elektrokoagulationen

Philipp, K 280

Allgemeine Richtlinien zur Betreuung ambulanter gynäkologischer Patientinnen nach operativen Eingriffen

Brehm, R 283

Praktische Erfahrungen mit Impfungen gegen unspezifischen Fluor vaginalis mit einer Laktobazillus-Vakzine

Müller, G.; Salzer, H 285

Autorenregister 288

Suppl. 1 Jahrestagung der Österreichischen Gesellschaft fur Gynäkologie und Geburtshilfe Juni 1982, Bad Ischl

Suppl. 2 Trichomoniasis

Referate des Symposiums über Frichomoniasis, Basel, 20. Oktober 1981

Suppl. 3 Schweizerische Gesellschaft für Gynäkologie und Geburtshilfe unter Mitwirkung der

Schweizerischen Gesellschaft für medizinische Genetik

Bericht über die Jahresversammlung, 24. bis 26. Juni 1982 in Zurich

Société Suisse de Gynécologie et Obstétrique avec la collaboration de la Société Suisse de génétique médicale

Rapport de ГAssemblée annuelle, 24 au 26 juin 1982 à Zurich 